\title{
TITLE: Bone morphogenetic protein 4 reduces global H3K4me3 to inhibit proliferation and promote differentiation of human neural stem cells
}

\section{RUNNING TITLE: BMP4 reduces H3K4me3 in human NSCs}

Sonali Nayak, ${ }^{1 *}$ Benjamin Best,${ }^{1 *}$ Emily Hayes,${ }^{1}$ Ashorne Mahenthiran, ${ }^{1}$ Nitin R Wadhwani, ${ }^{2}$ Barbara Mania-Farnell, ${ }^{3}$ Rintaro Hashizume, ${ }^{4}$ John A Kessler, ${ }^{6}$ Charles David James, ${ }^{4}$ Tadanori Tomita, ${ }^{1,4}$ Guifa Xi, ${ }^{1,4,5}$

${ }^{1}$ Falk Brain Tumor Center, Division of Pediatric Neurosurgery, ${ }^{2}$ Department of Pathology, Ann \& Robert H. Lurie Children's Hospital of Chicago, Northwestern University Feinberg School of Medicine, Chicago, IL 60611

${ }^{3}$ Department of Biological Sciences, Purdue University Northwest, Hammond, IN, 46323

${ }^{4}$ Department of Neurological Surgery, Northwestern University Feinberg School of Medicine, Chicago, IL 60611

${ }^{5}$ Developmental Biology Program, Stanley Manne Research Institute, Ann \& Robert H. Lurie Children's Hospital of Chicago, Northwestern University Feinberg School of Medicine, Chicago, IL 60611

${ }^{6}$ Department of Neurology, Northwestern Memorial Hospital, Northwestern University Feinberg School of Medicine, Chicago, IL 60611

*equal contribution of this work

\section{Corresponding author:}

Guifa Xi, MD, PhD

Affiliation: Division of Pediatric Neurosurgery, Ann \& Robert H. Lurie Children's Hospital of Chicago, Northwestern University Feinberg School of Medicine

Address: 225 E Chicago Ave, PO Box \#28, Chicago, IL 60611

Phone: (312)503-4296

Fax: (312)227-9679

E-mail: gxi@luriechildrens.org 
AUTHOR CONTRIBUTIONS: S.N, B.B, A.M, E.H, and G.X: collection and/or assembly of data; manuscript revision/editing; final approval of manuscript; N.R.W: autopsy human fetal brain sample preparation and collection of immunohistochemistry data; final approval of manuscript; B.M-F: conception and design; manuscript revision/editing; final approval of manuscript; R.H: conception and design, material sharing and data collection, final approval of manuscript; J.A.K, and C.D.J: conception and design, data analysis and interpretation, manuscript revision/editing, final approval of manuscript; T.T: conception and design; administrative and financial support, conception and design, final approval of manuscript; G.X: conception and design, collection and/or assembly of data, data analysis and interpretation, manuscript writing, final approval of manuscript.

ACKNOELEDGEMENTS: This project was partially supported by National Cancer Institute SPORE grant (P50CA221747-01A1) Career Enhancement Award and by the Rory David Deutsch Foundation, the Surgical Neuro-Oncology Research Fund of Ann \& Robert H. Lurie Children's Hospital (A\&RLCH) of Chicago, and the Dr. Ralph and Marian C. Falk Medical Research Trust.

We appreciate generosity of Dr. David G. Skalnik at Indiana University School of Medicine to provide rabbit polyclonal antibody against WDR82 and pcDNA3-WDR82-HA plasmid. We also thank Drs. Kunliang Guan and Cheryl Arrowsmith who created pcDNA3-HA and pET28-SETD1AMHL and its control pET28-MHL plasmids, respectively and donated to Addgene.

DISCLOSURE OF POTENTIAL CONFLICTS OF INTEREST: The authors declare no conflicts of interest. 


\section{ABSTRACT}

Posttranslational modifications (PTMs) on histone tails spatiotemporally dictate mammalian neural stem cell (NSC) fate. Bone morphogenetic protein 4 (BMP4), a member of the transforming growth factor $\beta$ (TGF- $\beta$ ) superfamily, suppresses NSC proliferation and fosters differentiation into astroglial cells. Whether PTMs mediate these effects of BMP4 is unknown. Here we demonstrate that BMP4 signaling causes a net reduction in cellular histone H3 lysine 4 trimethylation (H3K4me3), an active histone mark at promoters of genes associated with human NSC proliferation. We also show that H3K4me3 reduction by BMP4 is mediated by decreased expression of SETD1A and WDR82, two methyltransferase components of SETD1A-COMPASS. Down-regulation of these components decreases expression of key genes expressed in hNSCs, while ectopic expression via transfection dedifferentiates human astrocytes (HAs). These observations suggest that BMP4 influences NSC fate by regulating PTMs and altering chromatin structure.

KEY WORDS: Bone morphogenetic protein 4; self-renewal; proliferation; epigenetic; human neural stem cell

SIGNIFICANCE STATEMENT: BMP4 is critical in determining hNSC fate. Whether histone posttranslational modifications (PTM) mediate the effects of BMP4 is unknown. Here we report that H3K4me3, brought about by its methyltransferases SETD1A and WDR82, at promoters of stem cell genes OCT4 and NESTIN is involved in human neural stem cell (hNSC) maintenance. BMP4 promotes hNSC astroglial differentiation in part through reduction of SETD1A and WDR82 and thus decreased frequency of $\mathrm{H} 3 \mathrm{~K} 4 \mathrm{me} 3$ at the promoters of these genes. These results provide evidence that BMP4 promotes hNSC differentiation through a potential epigenetic mechanism and extend our understanding of the role of histone PTM in central nervous system development. 


\section{INTRODUCTION}

Neural stem cells (NSCs) either self-renew or differentiate into neurons, astrocytes and oligodendrocytes [1, 2]. Higher order chromatin structure precisely regulates gene expression to dictate NSC fate $[3,4]$. Histones are key components of chromatin and subject to a wide variety of dynamic post-translational modifications (PTMs) [5]. Histone and associated modifications are important determinants of human NSC self-renewal, proliferation and differentiation [4, 6-9].

Trimethylation of histone $\mathrm{H} 3$ lysine $4(\mathrm{H} 3 \mathrm{~K} 4 \mathrm{me} 3)$ by a complex of proteins associated with SET1A/B (SETD1A/B-COMPASS) [10-12], is pivotal in determining human NSC fate [13-17]. H3K4me3 is enriched near transcription start sites (TSSs) at the 5' regions of virtually all activelyexpressed genes; its presence has a strong positive correlation with gene transcription rates, polymerase II promoter occupancy [18, 19], and transcriptional consistency [20]. WDR82 is a specific subunit of SETD1A/B. Other subunits of SETD1A/B-COMPASS include WDR5 [21], DPY30 [22], and ASH2L [23].

Bone morphogenetic protein 4 (BMP4), a member of the TGF- $\beta$ superfamily, is essential for central nervous system (CNS) development due to its influence on NSC self-renewal, proliferation and differentiation [24]. BMP4 signaling affects the expression of key genes that affect NSC fate. For example, BMP4 increases CXXC finger protein 5 (CXXC5) to repress Wnt signaling and promote NSC differentiation [25]. It increases expression of inhibitor of differentiation 4 (ID4) and ID2, which inhibits oligodendroglial lineage commitment and promotes astrocyte commitment [26]. BMP4 inhibits mitogen-activated protein kinase (MAPK) pathways that support stem cell selfrenewal [27], and it stimulates msx2 and p21 to mediate pro-apoptotic effects on NPCs in the ventricular zone [28]. 
However, little is known regarding effects of BMP4 on chromatin structure and how these changes influence gene expression. BMP4 differentiates CD133-positive glioma stem cells to astrogliallike cells [29] and inhibits their proliferation through decreasing CYCLIN D1 expression [30]. SMAD-mediated BMP4 signaling regulates embryonic stem cell proliferation by altering H3K4me3 enrichment at promoters of proliferation genes [31]. In NSCs, the CYCLIN D1 promoter is enriched for H3K4me3 [20]. We found that Human StemPro® NSCs are CD133 positive which led to the hypothesis that BMP4 inhibits human StemPro® NSC proliferation through reduction of H3K4me3 at the CYCLIN D1 gene. In this study we show that BMP4 signaling regulates H3K4me3 and chromatin structure, thereby influencing expression of several genes with important consequences for NSC differentiation and proliferation.

\section{MATERIAL AND METHODS}

\section{Reagents and antibodies}

Knockout ${ }^{T M}$ DMEM/F-12(Cat\#12660-012), StemPro® neural supplement (Cat\#A10508-01), epidermal growth factor (EGF), fibroblast growth factor-2 (FGF-2), GlutaMAX ${ }^{\text {TM }}$-I CTS ${ }^{\text {TM }}$ (100X) (Cat\#A12860), Geltrex® hESC-qualified Reduced Growth Factor Basement Membrane Matrix (Cat\# A1413301), StemPro® Accutase® Cell Dissociation Reagent (Cat\#A11105), and Geltrex® LDEV-Free hESC-qualified Reduced Growth Factor Basement Membrane Matrix (Cat\#A14133) were purchased from Life Technologies (Frederick, MD, USA). Accutase was purchased from Millipore (Billerica, MA, USA). Heparin (Cat\#H3149) and ascorbic acid (Cat\#A8960) were from Sigma Aldrich (St. Louis, MO, USA).

Rabbit polyclonal antibody against WDR82 was a generous gift from Dr. David G. Skalnik (Indiana University School of Medicine, IN, USA). Antibodies were purchased from the following companies: BMP4 (sc-6896), OCT4 (sc-8629), and GAPDH (sc-25778) from Santa Cruz Biotechnology (Dallas, TX, USA); human SETD1A (Cat\#A300-289A) and SETD1B (Cat\#302- 
280A) from Bethyl Laboratories (Montgomery, TX, USA); H3K4me3 (Cat\#9727), Histone H3 (Cat\#9715), ASH2L (Cat\#5019); WDR5 (Cat\#13105), RBBP5 (Cat\#13171), and CYCLIN D1 (Cat\#2922) from Cell Signaling Technology; CD133 (ab5558), and $\beta$-actin (ab-8227) from Abcam (Cambridge, MA, USA).

\section{Immunohistochemistry}

Formalin-fixed, paraffin-embedded (FFPE) fetal brain tissue slides containing lateral ventricle lining from autopsy brain samples, from two pediatric patients who died of non-brain related diseases, were obtained from the Department of Pathology, Ann \& Robert H. Lurie Children's Hospital of Chicago. FFPE slides were de-paraffinized in xylene, and hydrated through a graded series of alcohols. Endogenous peroxidases were blocked with 3\% hydrogen peroxide. Antigen retrieval was performed by boiling for $20 \mathrm{~min}$ in a $0.01 \mathrm{M}$ sodium citrate $(\mathrm{pH} 6.0)$ solution and endogenous biotin was blocked using the Avidin/Biotin Blocking Kit (Vector Labs, SP-2001). Slides were incubated overnight with primary antibodies against anti-rabbit H3K4me3 (Cell Signaling Technology, Cat\#9727, 1:400). Following incubation with biotin-labeled secondary antibodies (Vector Laboratories BA1000), antigens were visualized with streptavidin-biotin (Vectastain Elite ABC kit; Vector Laboratories) followed by Vector $^{\circledR}$ NovaRED $^{\mathrm{TM}}$ (Vector Laboratories) and counterstained with hematoxylin (Richard-Allen Scientific). Images were captured on a Leica DMR-HC upright microscope and analyzed using OpenLab 5.0 software.

\section{Cell culture: primary neurospheres, spontaneously differentiated cells and human astrocytes}

Human StemPro® NSC line derived from fetal brain was purchased from Life Technologies, Inc. (Grand Island, NY, USA). For neurospheres, the cells were cultured in StemPro® NSC serum free medium (SFM), prepared with Knockout ${ }^{\mathrm{TM}}$ DMEM/F-12 medium including $2 \%$ StemPro® Neural Supplement, 20ng/ml bFGF, 20ng/ml EGF, 2mM GlutaMAX ${ }^{\mathrm{TM}}$-I supplement, 6 units/ml 
heparin and $200 \mu \mathrm{M}$ ascorbic acid. For routine passaging, cells were dissociated with StemPro® Accutase ${ }^{\circledR}$ Cell Dissociation Reagent. For spontaneous differentiation, cells were harvested as single cell suspensions with StemPro® Accutase ${ }^{\circledR}$ Cell Dissociation Reagent as per manufacturer's instruction. The cells were re-suspended in differentiation medium prior to plating on Geltrex® matrix-coated culture vessels, and grown with SFM without bFGF and EGF. The medium was changed after 2 days followed by replacement of half of the media every 2-3 days. Following 7 days of differentiation, all three neural lineages (astrocytes, neurons, and oligodendrocytes) were present. The normal human astrocyte cell line (HA) was kindly provided by Dr. Rintaro Hashizume and grown as described [32, 33].

\section{Immunofluorescence}

Seven days after primary neurospheres culture, cells were harvested as single cells and split with SFM or differentiation medium. For sphere staining, spheres were plated onto glass coverslips coated with Geltrex® matrix placed in individual wells of a 12-well culture plate in SFM and monitored under a microscope every $15 \mathrm{mins}$. Once spheres attached and began to differentiate they were fixed with $4 \%$ paraformaldehyde in PBS (Pierce Chemical Co., Rockford, IL). For staining of differentiated cells, cells were plated onto glass coverslips coated with Geltrex® matrix, and cultured in individual wells of a 12-well culture plate with SF differentiation medium. Seven days after plating, coverslips were fixed with $4 \%$ paraformaldehyde in PBS. Fixed cells were blocked with $10 \%$ donkey serum and $0.3 \%$ Triton X-100 in PBS and incubated with CD133 (1:100), OCT3/4 (1:100), SOX2 (1:100), and NESTIN (1:100) for neurospheres; and with MUSHASHI (1:100), GFAP (1:200), $\beta$-TUBULIN (1:100) for neurons, and O4 (1:100) for differentiated astrocytes, neurons and oligodendroglial cells. . Alexa Fluor 488 or cy3 labeled secondary antibodies (dilution 1:200) (Jackson Lab, ME, USA) were used for detection. Nuclei were counterstained with DAPI. Images were captured with a Leica DM-IRB inverted microscope and analyzed using OpenLab 5.0 software. 


\section{Constructs and transfections}

pcDNA3-WDR82-HA was a generous gift from Dr. David G. Skalnik (Indiana University School of Medicine, IN, USA). pcDNA3-HA was a gift from Kunliang Guan (Addgene plasmid \#13512). Human His-tagged SETD1A expression pET28-SETD1A-MHL plasmid and its control pET28MHL were gifts from Cheryl Arrowsmith (Addgene plasmid \# 32868 and \#26096, respectively). Human short interference RNA (siRNA) against human SETD1A (Gene ID 9739, Cat\# SR306505), short hairpin RNA (shRNA) against WDR82 (Gene ID 80335, Cat\#TG301034), and scrambled control shRNA cassette in pGFP-V-RS Vector (Cat\#TR30013) were purchased from Origene (Rockville, MD, USA). Lipofectamine ${ }^{\mathrm{TM}}$ stem transfection reagent (Cat\#STEM00003) for cDNA cloning and shRNA, and lipofectamine RNAiMAX (Cat\#13778030) for siRNA transfection were purchased from Life technologies (Carlsbad, CA, USA).

For SETD1A and WDR82 knock-down in hNSCs (StemPro®), sphere hNSCs were treated with WDR82 shRNA or its scrambled shRNA control (scrCtrl) using Lipofectamine ${ }^{\mathrm{TM}}$ stem transfection reagent or with 30nM hSETD1A and control siRNAs using lipofectamine RNAiMAX as per manufacturer's instructions. For human SETD1A and WDR82 over-expression in normal HAs, HAs were plated in 6-well plates and transiently transfected with pET28-MHL or pET28-SETD1AMHL, or with pcDNA3-WDR82-HA or pcDNA-HA using Lipofectamine ${ }^{\mathrm{TM}}$ stem transfection reagent as per manufacturer's instructions. Transfected cells were subjected to sphere formation assays or lysed after $48 \mathrm{~h}$ for quantitative real-time PCR, chromatin immunoprecipitation PCR and western blots.

\section{Neurosphere formation, cell proliferation and differentiation assays}

Human StemPro® NSCs were harvested as single cells and plated on 12 -well plates at $8.33 \times$ $10^{4}$ cells/well and treated with $100 \mathrm{ng} / \mathrm{ml} \mathrm{BMP4}$, or with buffer. Sphere formation was monitored daily and the number of spheres was counted 4 days after treatment. At 4 days the spheres were 
centrifuged and dissociated with StemPro® Accutase ${ }^{\circledR}$ into single cells. The cells were resuspended in $1 \mathrm{ml} \mathrm{NSC} \mathrm{SFM} \mathrm{and} \mathrm{counted} \mathrm{with} \mathrm{a} \mathrm{hemocytometer.} \mathrm{Sphere} \mathrm{and} \mathrm{cell} \mathrm{numbers} \mathrm{from}$ triplicate wells were counted and analyzed. For sphere differentiation assays, human StemPro® NSCs were harvested as single cells and plated on 6 -well plates at a density of $4.5 \times 10^{5}$ cells/well. Sphere formation was monitored daily. On day 4 after plating, the spheres were treated with 100ng/ml BMP4, or buffer. Sphere differentiation was investigated daily for 7 days and cells were harvested for RNA extraction for PCR array assays.

For HA sphere formation assays, the cells were plated in 6-well plates and transfected with pET28-hSETD1A-MHL and pcDNA3-WDR82-HA using StemPro® NSC transfection reagent. After 48 hrs, the culture medium was replaced with Neurobasal medium supplement with N2, B27, EGF $20 \mathrm{ng} / \mathrm{ml}$ and bFGF $20 \mathrm{ng} / \mathrm{ml}$. At day 4, sphere numbers from a minimum of ten fields in triplicate wells were counted and analyzed.

Sphere or cell numbers were averaged and graphed with Graphpad Prism 7.0 software (La Jolla, CA, USA). $P$ values were calculated using 2 -sided Student's $t$ test, with $p<0.05$ considered significant.

\section{Quantitative real-time PCR}

Total RNA was isolated with the RNeasy Mini Kit (Qiagen, Valencia, CA, USA) from primary sphere cultures and spontaneously differentiated Human StemPro® NSCs, treated with BMP4 and from non-treated controls. cDNA was synthesized with qScript cDNA SuperMix $(5 \times)$ (Quanta Biosciences, 95048-025) followed by real-time (RT) PCR with primers as indicated (Supplementary Table 1). To ensure accuracy, an internal reference reaction using GAPDH was performed on the same sample as used for the target gene. The results were standardized with the formula: $\triangle \mathrm{CT}=\mathrm{CT}_{\text {Ref }}-\mathrm{CT}_{\text {Target }}$ and converted to fold change of target gene over reference 
gene $\left(F=2^{-\Delta C T}\right)$. Data from a minimum of 3 independent experiments were used to quantify gene expression. $\mathrm{P}$ values of less than 0.05 were considered statistically significant.

\section{Protein extraction and western blots}

Total protein was extracted with RIPA buffer (Cell Signaling Technology, Cat\#9806) with proteinase (Cell Signaling Technology, Beverly, MA, USA), phosphatase (Sigma) inhibitor cocktails and phenylmethylsulfonyl fluoride (PMSF, Roche). Total histone was extracted using a histone extraction kit (ab113476, Abcam) as per manufacturer's instructions. Protein concentrations were quantified with the BCA Protein Assay Kit (Cat\#23227, Thermo Fisher Scientific Inc.) with a Nanodrop ND-1000 (Thermo Fisher Scientific Inc.). Equal amounts of cell lysate or concentrated conditioned medium were resolved by sodium dodecyl sulfatepolyacrylamide gel electrophoresis and transferred to nitrocellulose membranes (Bio-Rad, Hercules, CA, USA). The membrane was blocked for $60 \mathrm{~min}$ with $5 \%$ non-fat dry milk in Trisbuffered saline and Tween 20 (1:1000), followed by blotting with primary antibodies overnight at $4^{\circ} \mathrm{C}$. Primary antibodies included: polyclonal anti-goat BMP4 (1:500), OCT3/4 (1:200), H3K4me3 (1:1000), H3 (1:2500), CYCLIN D1 (1:1000), ß-ACTIN (1:3000), GAPDH (1:2000); and antihuman SETD1A (A300-289A, 1:1000) from Bethyl Laboratories. After washing with Tris-buffered saline and Tween 20, membranes were incubated for $1 \mathrm{~h}$ at room temperature with horseradish peroxidase conjugated donkey anti-rabbit antibody (sc-2305, 1:5000), donkey anti-mouse antibody (sc-2306, 1:5000), or donkey anti-goat antibody (sc-2020, 1:5000) and signal was detected with enhanced chemiluminescence substrate (Bio-Rad).

\section{Chromatin immunoprecipitation (ChIP)}

ChIP was performed according to the manufacturer's instructions (Abcam). Briefly, $1 \times 10^{6}$ cells were washed with ice cold PBS with proteinase inhibitor cocktail prior to fixation in $1 \%$ formaldehyde in PBS at room temperature for $10 \mathrm{~min}$ and quenched with $0.125 \mathrm{M}$ glycine for 5 


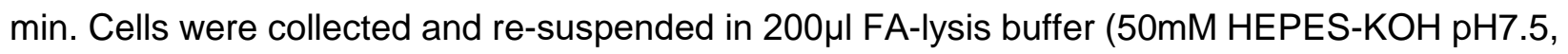
140mM NaCl, $1 \mathrm{mM}$ EDTA pH8.0, 1\% Triton X-100, 0.1\% Sodium Deoxycholate, $0.1 \%$ SDS, $1 \mathrm{mM}$ PMSF, $1 \mu \mathrm{g} / \mathrm{mL}$ leupeptin, $1 \mu \mathrm{g} / \mathrm{mL}$ pepstatin) and disrupted using a $251 / 2$ gauge syringe while on ice. The lysate was transferred into a $5 \mathrm{ml}$ conical tube and diluted with $1.3 \mathrm{ml}$ dilution buffer $(0.01 \%$ SDS, $1.1 \%$ Triton X-100, 1.2mM EDTA pH 8.0, 16.7mM Tris-HCl pH 8.0, 167mM NaCl), sonicated and processed according to manufacturer's protocol. Rabbit polyclonal H3K4me3 antibody (Cell Signaling Technology, Cat\# 9727) and IgG from rabbit serum (Cell Signaling Technology, Cat\# 2729) were used to collect immunoprecipitated DNA fragments. All DNA fragments were cleanedup with GenElute ${ }^{\mathrm{TM}}$ PCR Clean-Up Kit (Sigma Aldrich Co., St. Louis, MO, USA) prior to PCR analysis. Human OCT4 (Cell Signaling Technology, Cat\#4641) and CCND1 (Cell Signaling Technology, Cat\#12531) and NESTIN (Cat\# GPH1015041 (-) 01A, Qiagen, Germantown, MD, USA) promoter primers were used following real-time PCR analysis.

\section{RESULTS}

\section{H3K4me3 is a stem marker for human NSCs}

Slides from the region lining the lateral ventricle were prepared from two human fetal brain samples and immunostained for H3K4me3. H3K4me3 positive cells were enriched in the SVZ (Figure 1A), in agreement with previous findings from rodents [34] and baboons [13]. To determine if H3K4me3 and its methyltransferases hSETD1A and WDR82 were altered upon differentiation, RNA, total protein, and histones were extracted from spheres and from differentiated human NSCs (StemPro®). Real-time PCR and western blots both showed that hSETD1A and WDR82 expression was higher in sphere cultured cells compared to differentiated cells (Figure 1C and D). Other proteins included in the hSETD1A-COMPASS complex were not significantly changed (Supplementary Figure 1). H3K4me3 was readily detected in undifferentiated sphere-cultured hNSCs, but was undetectable in differentiated cells (Figure 1D). 
These results indicate that $\mathrm{H} 3 \mathrm{~K} 4 \mathrm{me} 3$ and the activity of its methyltransferases hSETD1A and WDR82 are associated with NSC self-renewal, but not with differentiated cells.
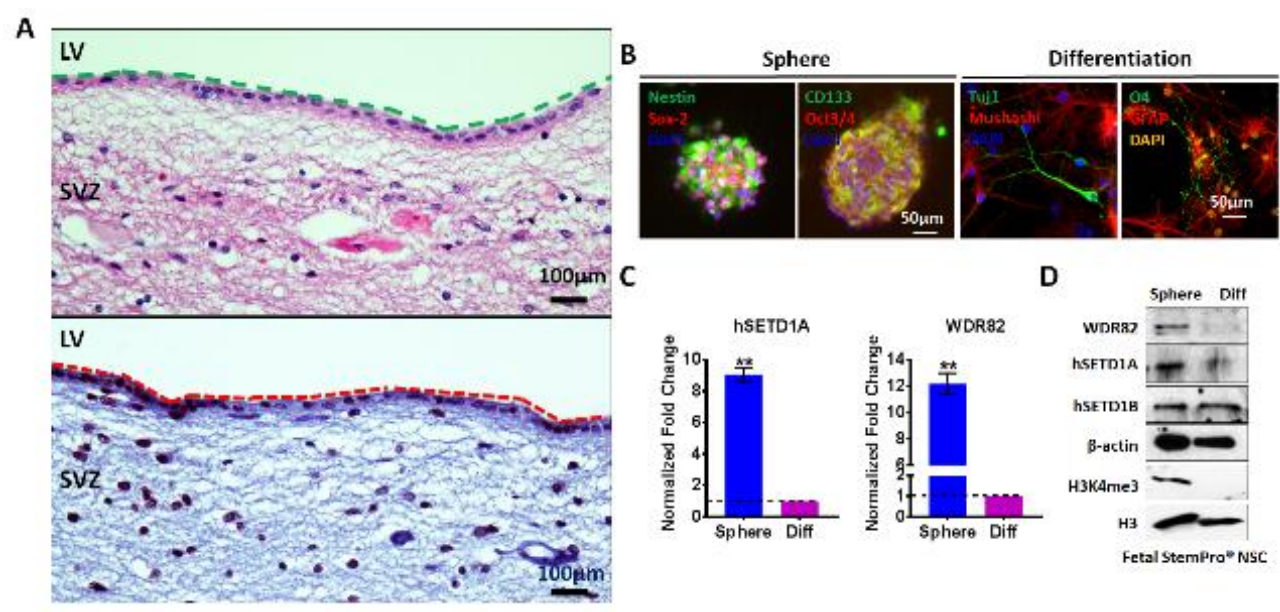

Figure $1 \mathrm{H} 3 \mathrm{~K} 4 \mathrm{me} 3$ is present in human NSCs undergoing self-renewal but not in differentiated cells. A. Gross anatomy of partial fetal SVZ architecture and localization of H3K4me3 positive cells. View of fetal brain in the area of the lateral ventricle (LV), showing the SVZ with H3K4me3 positive cells. Top panel: H\&E staining (LV and SVZ are separated by a green dashed line). Bottom panel: immunostaining with H3K4me3 antibody (LV and SVZ are separated by a red dashed line). B. Immunofluorescence of human StemPro® NSC neurospheres positive for NESTIN, SOX2, CD133 and OCT4 and differentiated cells positive for MUSHASHI, GFAP, TUJ1 and O4. C. Real-time PCR showing hSETD1A and WDR82 expression in spheres and differentiated human StemPro® NSCs. D. Western blots using total protein extracts and histone from un- (UD) and -differentiated (Diff) cells. Error bars show the standard error of three independent experiments $\left({ }^{* *} p<0.01\right)$.

\section{H3K4me3 promoter occupancy determines hNSC fate}

We examined the expression of OCT4 [35], a crucial stem cell self-renewal marker, and NESTIN, a neural stem/progenitor marker, for potential relationships with H3K4 methylation. Both were expressed in NSCs, with decreased expression in spontaneously differentiated cells, (Figure 2 AE). Using H3K4me3 ChIP coupled with real-time PCR (Figure 2F) we determined that H3K4me3 
is enriched at OCT4 and NESTIN promoters in undifferentiated hNSCs relative to their differentiated derivatives (Figure 2F).



Figure 2 H3K4me3 is present at promoters of genes associated with NSC self-renewal. A. Experimental design for sample collection. B-E Real-time PCR (B and D) and western blots ( $C$ and $E)$ showing OCT4 and NESTIN mRNA and protein in undifferentiated spheres (Sphere) and spontaneously differentiated (Diff) human StemPro® NSCs. F. Chromatin-immunoprecipitation (ChIP) with rabbit IgG and H3K4me3 and detected with real-time PCR using promoter primers. Error bars show the standard error of three independent experiments $\left({ }^{*} p<0.05,{ }^{* *} p<0.01\right)$.

\section{BMP4 decreases H3K4me3 at CCND1 promoter in differentiated hNSCs}

To determine whether BMP4 inhibits human StemPro® NSCs proliferation through reduction of H3K4me3 at the CYCLIN D1 gene, hNSC neurospheres were cultured in 100ng/ml BMP4 (Figure $3 A$ ), which reduced both the number of neurospheres (diameter $>250 \mu \mathrm{m}$ ) (Figure $3 \mathrm{~B}$ and $\mathrm{C}$ ) and the overall number of cells (Figure 3D). BMP4 reduced CYCLIN D1 expression (Figure 3F and G) and CYCLIN D1 promoter H3K4me3 occupancy (Figure 3H). 



C

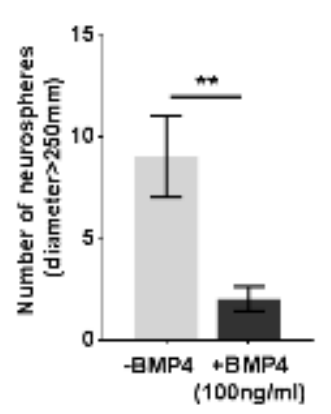

D


G

$$
\begin{aligned}
& \text { Spheres formation assay } \\
& \text { Cell counts } \\
& \text { Real-time PCR } \\
& \text { Western blots }
\end{aligned}
$$

CCND1

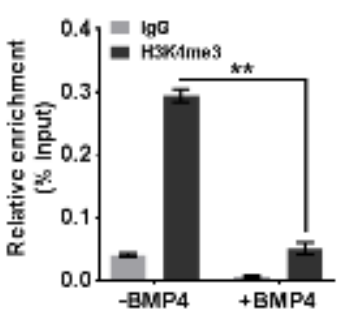

Figure 3 BMP4 inhibits human neurosphere formation and decreases H3K4me3 at the CYCLIN D1 promoter A. Experimental design. B, C and D. Representative images showing sphere morphology (B), number of neurospheres (diameter $>250 \mu \mathrm{m}$ ) (C) and cell numbers (D) at day 4 . E. Western blots showing H3K4me3 levels. E and F. Real-time PCR (E) and western blot (F) for CYCLIN D1. G. Chromatin-immunoprecipitation (ChIP) with rabbit IgG and H3K4me3 combined with real-time PCR to detect CYCLIN D1 promoter. Error bars show the standard error of three independent experiments ${ }^{* *}$ $\mathrm{p}<0.01)$.

\section{BMP4 reduces H3K4me3 at the NESTIN promoter and promotes hNSC differentiation}

We monitored differentiation of neurospheres from hNSCs following treatment with 100ng/ml BMP4 vs untreated controls (Figure 4A). By the fourth day nearly all neurospheres treated with BMP4 had attached and migrated (Figure 4B). Additionally, H3K4me3 markedly decreased in the treatment group compared to the controls (Figure 4C). NESTIN expression, examined by real- 
time PCR (Figure 4D) and western blot (Figure 4E), showed significant decreases with BMP4 treatment, as did levels of $\mathrm{H} 3 \mathrm{~K} 4 \mathrm{me} 3$ at the NESTIN promoter (Figure 4F).

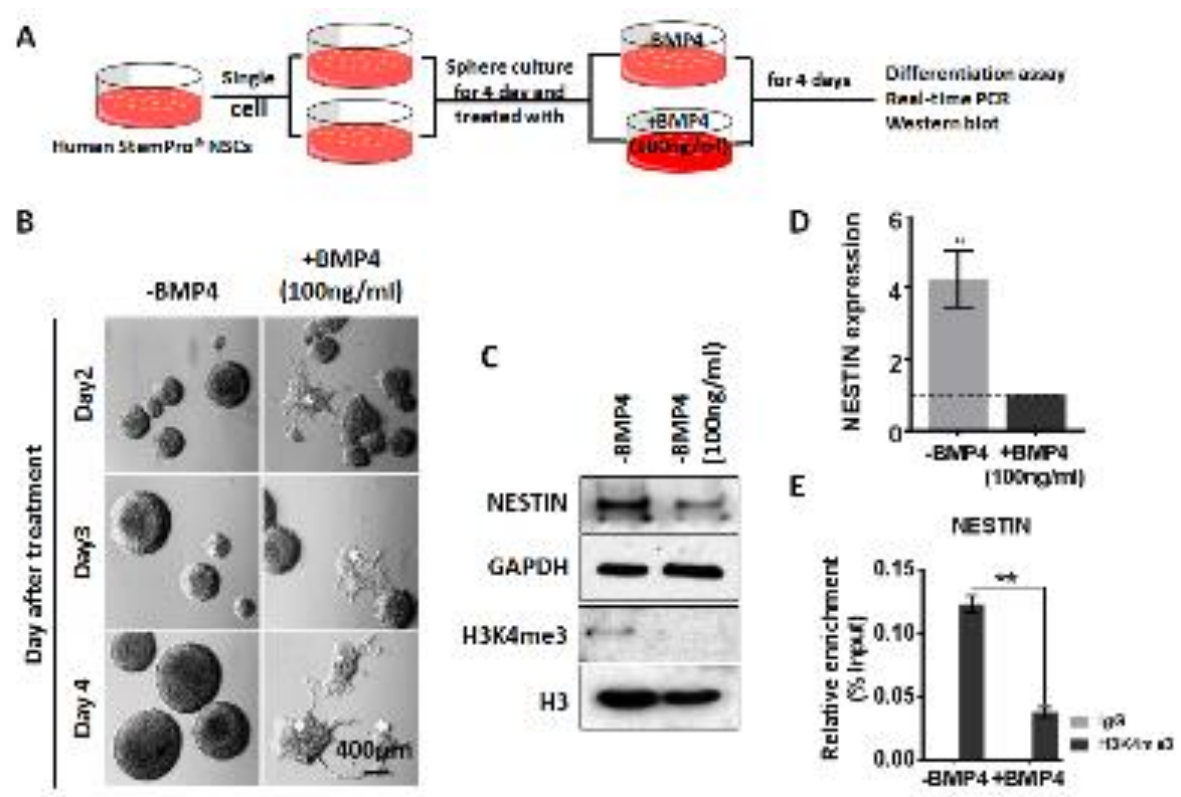

Figure 4 BMP4 drives human neurosphere differentiation with reduction of NESTIN promoter H3K4me3 occupancy. A. Experimental design. B. Representative images showing sphere morphology +BMP4 100ng/ml, in comparison to control (-BMP4). C. Western blots using total and histone extracts to detect NESTIN and H3K4me3, GAPDH and H3 as controls. D. Real-time PCR shows expression of NESTIN. E. Chromatin-immunoprecipitation (ChIP) with rabbit IgG and H3K4me3 combined with realtime PCR using NESTIN promoter primers. Error bars show the standard error of three independent experiments. $\left({ }^{* *} p<0.01\right)$

\section{BMP4 reduces SETD1A and WDR82 expression, with a corresponding reduction in}

\section{cellular H3K4me3}

BMP4 reduced levels of WDR82 and human SETD1A levels (Figure 5A). To determine the biologic effects in NSCs of reduced SETD1A and WDR82, hNSCs were incubated with SETD1A siRNA or transduced with lentivirus expressing WDR82 shRNA. Both treatments decreased sphere numbers in comparison to controls (Figure 5B). Target transcripts as well as transcripts associated with stemness (OCT4 and NESTIN) and proliferation (CCND1) also decreased (Figure 5C and D). H3K4me3 chromatin immunoprecipitation coupled with real-time PCR revealed that 
these transcript reductions occurred in conjunction with decreased H3K4me3 promoter occupancy (Figure 5E).

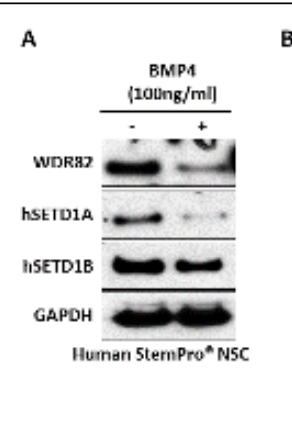

B

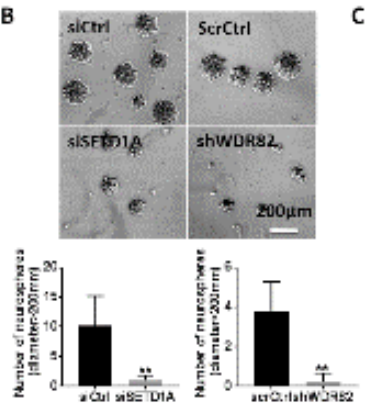

C HSETDIA WDR62 CCNDI NESTIN OCT4



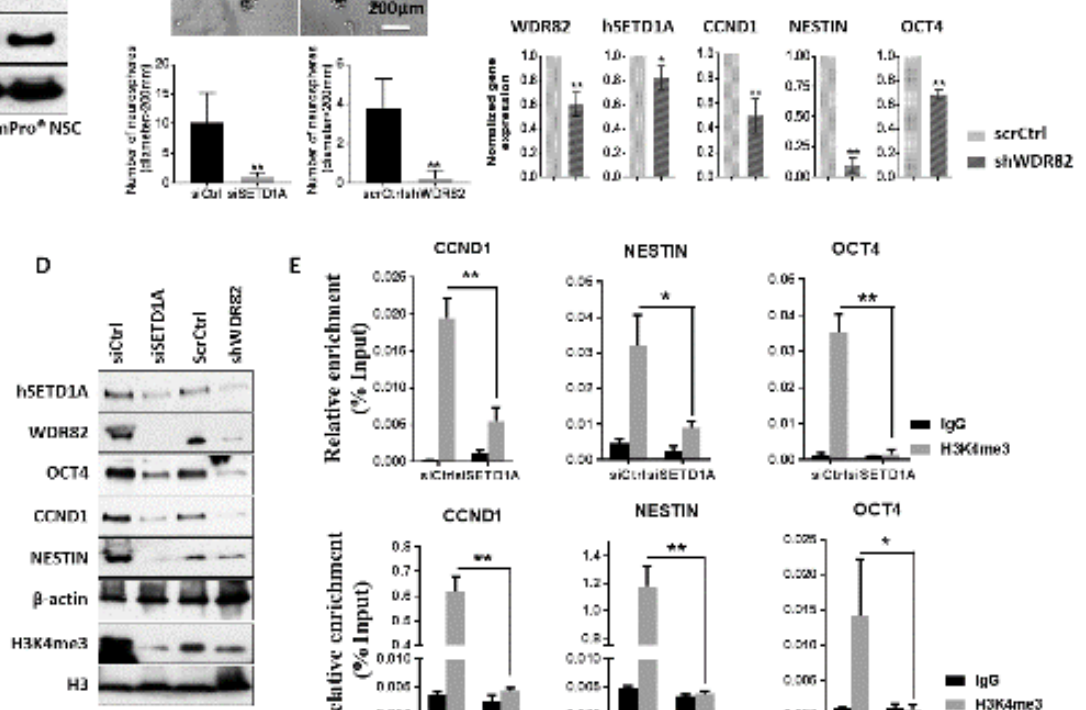

E
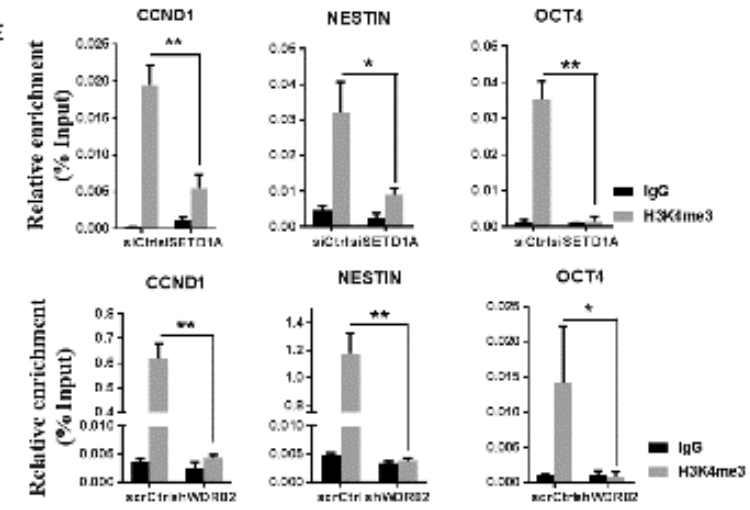

Figure 5 BMP4 reduces global H3K4me3 promoter occupancy through reduction of its methyltransferases WDR82 and hSETD1A. A. Western blots shows WDR82 and human SETD1A/B expression following 100 $\mathrm{ng} / \mathrm{ml}$ BMP4 treatment. B. Representative images and quantitative graphs showing sphere formation in human StemPro® NSCs following treatment with siRNA for hSETD1A (siSETD1A) or shRNA for WDR82 (shWDR82) vectors versus controls (control siRNA, siCtrl and scrambled shRNA, scrCtrl). C and D. Real-time PCR (C) and western blots (D) showing expression of hSETD1A, WDR82, OCT4, CCND1 and NESTIN, following treatments as in B. E. Real-time PCR using DNA from ChIP with rabbit IgG and H3K4me3 and detected with promoter primers for OCT4, CCND1 and NESTIN following treatment with siSETD1A, shWDR82 or siCtrl, scrCtrl, in StemPro® NSCs. Error bars show the standard error of three independent experiments. $\left({ }^{*} p<0.05,{ }^{* *} p<0.01\right)$

Ectopic expression of SETD1A and WDR82 increases expression of stemness and proliferation genes in normal human astrocytes

We investigated whether increasing expression of WDR82 or hSETD1A in human astrocytes (HAs) promotes cellular dedifferentiation. When astrocytes were transfected with WDR82 or hSETD1A cDNA, and cultured in hNSC medium for 4 days, sphere numbers increased (Figure 6A), as did expression of OCT4, CCND1 and NESTIN, indicated by real-time PCR and western 
bioRxiv preprint doi: https://doi.org/10.1101/2020.01.22.915934; this version posted January 23, 2020. The copyright holder for this preprint (which was not certified by peer review) is the author/funder, who has granted bioRxiv a license to display the preprint in perpetuity. It is made available under aCC-BY-NC-ND 4.0 International license.

blot analysis. (Figure $6 \mathrm{~B}$ and C). H3K4me3 promoter occupancy at these genes also increased (Figure 6D).
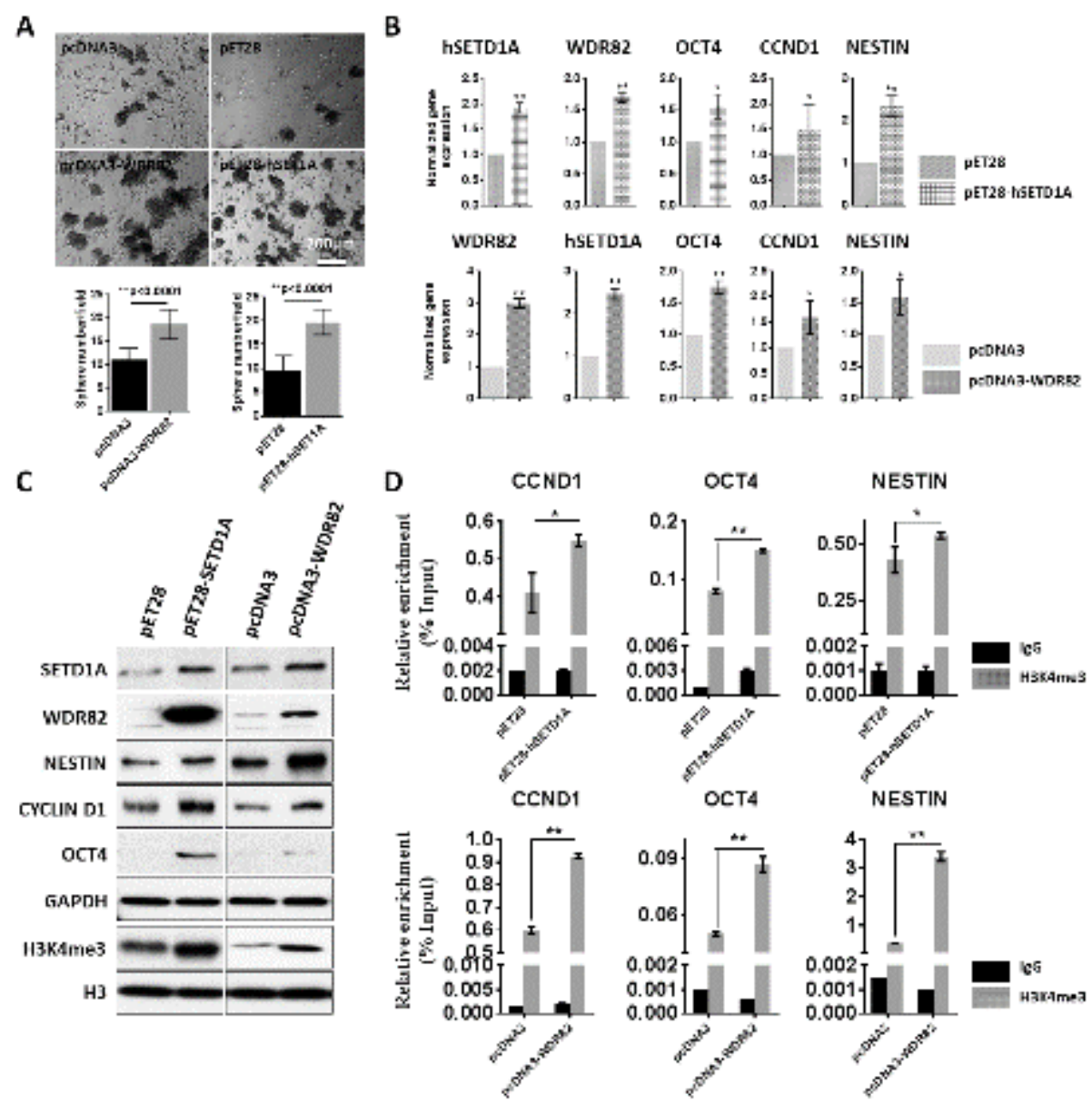

Figure 6 Ectopic expression of human SETD1A and WDR82 increases global H3K4me3 promoter occupancy in normal human astrocytes (HAs). A. Representative images and quantitative graphs show sphere formation from HAs following transfection with WDR82 (pcDNA3-WDR82) or human SETD1A ( $p E T 28-h S E T D 1 A-M H L)$ expression plasmids, in comparison to controls (pcDNA3 and pET28MHL). B and C. Real-time PCR (B) and western blots (C) show expression of hSETD1A, WDR82, OCT4, CCND1 and NESTIN. D. ChIP with rabbit IgG and H3K4me3 combined with real-time PCR using promoter primers for OCT4, CCND1 and NESTIN following HA transfection. Error bars show the standard deviation of three independent experiments. $\left({ }^{*} p<0.05,{ }^{* *} p<0.01\right)$ 


\section{DISSCUSSION}

BMP4 is critical for nervous system development due to its influence over several biological processes, ranging from dorsoventral patterning of the embryonic neural tube to proliferation, apoptosis, neurogenesis, and gliogenesis at different stages and locations [36-38]. BMP4 also plays important roles in the regulation of neural progenitor cell fate and promotes neuronal precursor differentiation in the spinal cord [39] and cortex [40]. Here we found that cellular H3K4me3 and its methyltransferases hSETD1A and WDR82 decrease upon BMP4-induced hNSC differentiation. This differentiation is accompanied by decreased expression of stem cell signature proteins OCT4 and NESTIN, in conjunction with decreased H3K4me3 at their promoters. We also showed that BMP4 reduces proliferation of human NSCs and inhibits neurosphere formation, this may be through decreased CYCLIN D1 promoter H3K4me3 occupancy, and thus CYCLIN D1 expression. Conversely, increased expression of WDR82 and hSETD1A in normal HAs increased global H3K4me3 levels and promoter occupancy at genes associated with stemness, proliferation and differentiation of hNSCs. Collectively these findings clearly demonstrate that hSETD1A and WDR82 are critical for maintaining neural stem cell populations, and decreasing in their expression promotes cellular differentiation.

Recent studies have shown histone PTMs are essential in hNSC self-renewal and differentiation. For example, H3K4me3 is present in undifferentiated progenitor cells within the SVZ and it is associated with distinct neurogenesis networks and pathways [13]. H3K4me3 is also required to ensure transcriptional precision at key genes during neural progenitor cell differentiation [20]. In the present study, we show that BMP4 changes H3K4me3 levels at specific loci including those involved with stemness (OCT4 and NESTIN) and proliferation CCND1 (Figures 2-4).

Mammalian SETD1A/B-COMPASS are responsible for H3K4me3 [41], with SETD1A most relevant in the development and maintenance of hNSCs [42, 43]. In silico analysis results from 
several published databases (GSE55379, GSE53404, and GSE43382) show high levels of SETD1A in human NSCs, with decreased levels in differentiated cells (Supplementary Figure 2A). In this study we show that SETD1A is high in human NSCs and decreases in differentiated cells (Figure 1C and D), in agreement with previous findings.

Our results show that expression of WDR82, a subunit of SETD1A-COMPASS, is also high in human NSCs, and that it decreases in differentiated cells (Figure 1C and D). WDR82 mediates alterations in $\mathrm{H} 3 \mathrm{~K} 4 \mathrm{me} 3$ levels and in so doing influences hNSC proliferation and apoptosis during normal embryonic growth and development [44]. Analysis of results from databases GSE15209 and GSE43382 show that WDR82 is high in human NSCs, but decreases in differentiated brain cortex (GSE15209) and differentiated human NSCs (GSE43382) (Supplementary Figure 2B). This is consistent with our results and highlights WDR82 involvement in regulating hNSC stemness and differentiation.

SETD1A acts through several mechanisms to bring about intracellular changes. Studies have shown that it modulates cell cycle progression through induction of a miRNA network to suppress P53 in human cancers $[45,46]$, independent of H3K4me3. A non-catalytic domain, "FLOS" (functional location on SETD1A), interacts with CYCLIN K and regulates DNA damage response gene expression [47]. In the present study, we found reduction of WDR82 decreased hSETD1A expression in hNSCs (Figure 5C and D), while ectopic expression of WDR82 markedly increased SETD1A (Figure 6B and C) in normal HAs, consistent with findings from other groups [48, 49]. Conversely, reduction of hSETD1A decreased hNSC WDR82 expression (Figure 5C and D), whereas induction of SETD1A markedly increased WDR82 in normal HAs (Figure 6B and C). WDR82 interacts with an RNA recognition motif (RRM) [48, 49], consisting of 95 highly conserved 
amino acids, at the N-terminal of hSETD1A protein (Supplementary Figure 3). Our findings indicate that WDR82 and hSETD1A control cellular H3K4me3 in hNSCs and suggest that BMP4 promotes hNSC differentiation through an epigenetic mechanism.

\section{CONCLUSION}

In this work, we have shown that H3K4me3 promoter occupancy mediated by hSETD1A and WDR82 is critical for hNSC fate. BMP4 decreases hSETD1A and WDR82, resulting in the reduction of H3K4me3 at key genes such as OCT4, NESTIN and CCND1 to inhibit proliferation and promote differentiation of hNSCs (Figure 7). These results extend our understanding of the role of histone PTMs in normal CNS development.

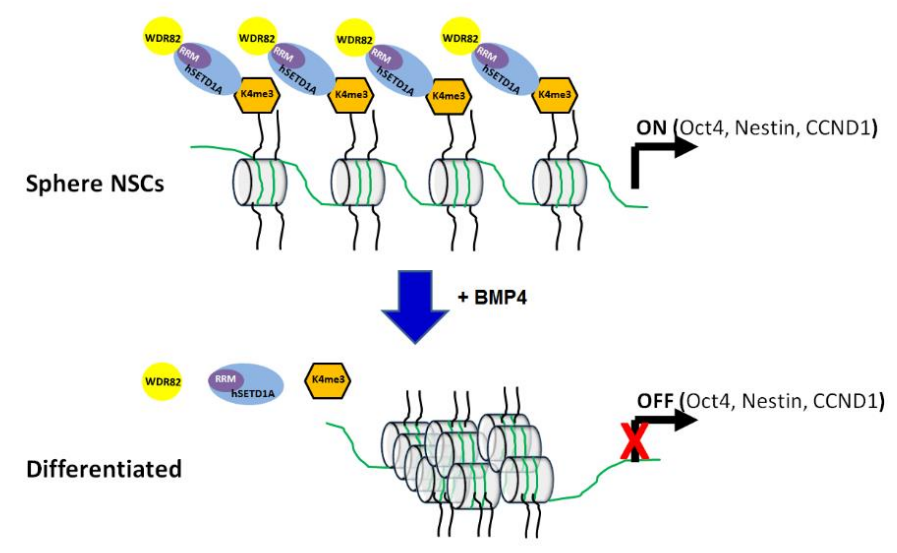

Figure 7. Schematic diagram showing that BMP4 decreases levels of H3K4me3 mediated by hSETD1A-WDR82 at promoters of key regulators to promote differentiation and inhibit proliferation of human neural stem cells (NSCs). 


\section{REFERENCES}

1. Zhao C, Deng W, Gage FH. Mechanisms and Functional Implications of Adult Neurogenesis. Cell. 2008;132:645-660.

2. Fasano CA, Dimos JT, Ivanova NB et al. shRNA knockdown of Bmi-1 reveals a critical role for p21-Rb pathway in NSC self-renewal during development. Cell stem cell. $2007 ; 1: 87-99$.

3. Zhou VW, Goren A, Bernstein BE. Charting histone modifications and the functional organization of mammalian genomes. Nature reviews Genetics. 2011;12:7-18.

4. Mohamed Ariff I, Mitra A, Basu A. Epigenetic regulation of self-renewal and fate determination in neural stem cells. J Neurosci Res. 2012;90:529-539.

5. Khorasanizadeh S. The nucleosome: from genomic organization to genomic regulation. Cell. 2004;116:259-272.

6. Ming GL, Song H. Adult neurogenesis in the mammalian brain: significant answers and significant questions. Neuron. 2011;70:687-702.

7. Juliandi B, Abematsu M, Nakashima K. Epigenetic regulation in neural stem cell differentiation. Development, Growth \& Differentiation. 2010;52:493-504.

8. Juliandi B, Abematsu M, Nakashima K. Chromatin remodeling in neural stem cell differentiation. Curr Opin Neurobiol. 2010;20:408-415.

9. Coskun V, Tsoa R, Sun YE. Epigenetic regulation of stem cells differentiating along the neural lineage. Curr Opin Neurobiol. 2012;22:762-767.

10. Bhaumik SR, Smith E, Shilatifard A. Covalent modifications of histones during development and disease pathogenesis. Nature structural \& molecular biology. 2007;14:1008-1016.

11. Glaser S, Schaft J, Lubitz S et al. Multiple epigenetic maintenance factors implicated by the loss of MII2 in mouse development. Development. 2006;133:1423-1432. 
12. Lee $\mathrm{JH}$, Tate $\mathrm{CM}$, You JS et al. Identification and characterization of the human Set1B histone H3-Lys4 methyltransferase complex. J Biol Chem. 2007;282:13419-13428.

13. Sandstrom RS, Foret MR, Grow DA et al. Epigenetic regulation by chromatin activation mark H3K4me3 in primate progenitor cells within adult neurogenic niche. Sci Rep. $2014 ; 4: 5371$.

14. He XB, Yi SH, Rhee YH et al. Prolonged membrane depolarization enhances midbrain dopamine neuron differentiation via epigenetic histone modifications. Stem Cells. $2011 ; 29: 1861-1873$.

15. Schmitz SU, Albert M, Malatesta $\mathrm{M}$ et al. Jarid1b targets genes regulating development and is involved in neural differentiation. EMBO J. 2011;30:4586-4600.

16. Wynder C, Stalker L, Doughty ML. Role of H3K4 demethylases in complex neurodevelopmental diseases. Epigenomics. 2010;2:407-418.

17. Gu B, Lee MG. Histone H3 lysine 4 methyltransferases and demethylases in self-renewal and differentiation of stem cells. Cell \& bioscience. 2013;3:39.

18. Bernstein BE, Kamal M, Lindblad-Toh K et al. Genomic maps and comparative analysis of histone modifications in human and mouse. Cell. 2005;120:169-181.

19. Schneider R, Bannister AJ, Myers FA et al. Histone H3 lysine 4 methylation patterns in higher eukaryotic genes. Nature cell biology. 2004;6:73-77.

20. Benayoun Bérénice A, Pollina Elizabeth A, Ucar D et al. H3K4me3 Breadth Is Linked to Cell Identity and Transcriptional Consistency. Cell. 2014;158:673-688.

21. Ang YS, Tsai SY, Lee DF et al. Wdr5 mediates self-renewal and reprogramming via the embryonic stem cell core transcriptional network. Cell. 2011;145:183-197.

22. Jiang $\mathrm{H}$, Shukla A, Wang $\mathrm{X}$ et al. Role for Dpy-30 in ES cell-fate specification by regulation of H3K4 methylation within bivalent domains. Cell. 2011;144:513-525. 
23. Wan $\mathrm{M}$, Liang $\mathrm{J}$, Xiong $\mathrm{Y}$ et al. The trithorax group protein Ash2l is essential for pluripotency and maintaining open chromatin in embryonic stem cells. J Biol Chem. 2013;288:5039-5048.

24. Xi G, Best B, Mania-Farnell B et al. Therapeutic Potential for Bone Morphogenetic Protein 4 in Human Malignant Glioma. Neoplasia (New York, NY). 2017;19:261-270.

25. Andersson T, Sodersten E, Duckworth JK et al. CXXC5 is a novel BMP4-regulated modulator of Wnt signaling in neural stem cells. J Biol Chem. 2009;284:3672-3681.

26. Samanta J, Kessler JA. Interactions between ID and OLIG proteins mediate the inhibitory effects of BMP4 on oligodendroglial differentiation. Development. 2004;131:4131-4142.

27. Qi X, Li TG, Hao J et al. BMP4 supports self-renewal of embryonic stem cells by inhibiting mitogen-activated protein kinase pathways. Proc Natl Acad Sci U S A. 2004;101:60276032.

28. Israsena N, Kessler JA. Msx2 and p21(CIP1/WAF1) mediate the proapoptotic effects of bone morphogenetic protein-4 on ventricular zone progenitor cells. J Neurosci Res. 2002;69:803-809.

29. Piccirillo SG, Reynolds BA, Zanetti N et al. Bone morphogenetic proteins inhibit the tumorigenic potential of human brain tumour-initiating cells. Nature. 2006;444:761-765.

30. Zhou Z, Sun L, Wang Y et al. Bone morphogenetic protein 4 inhibits cell proliferation and induces apoptosis in glioma stem cells. Cancer Biother Radiopharm. 2011;26:77-83.

31. Fei T, Xia K, Li Z et al. Genome-wide mapping of SMAD target genes reveals the role of BMP signaling in embryonic stem cell fate determination. Genome Res. 2010;20:36-44.

32. Hashizume R, Andor N, Ihara $\mathrm{Y}$ et al. Pharmacologic inhibition of histone demethylation as a therapy for pediatric brainstem glioma. Nat Med. 2014;20:1394-1396.

33. Chan KM, Fang D, Gan $\mathrm{H}$ et al. The histone H3.3K27M mutation in pediatric glioma reprograms H3K27 methylation and gene expression. Genes Dev. 2013;27:985-990. 
34. Lim DA, Huang YC, Swigut $T$ et al. Chromatin remodelling factor Mll1 is essential for neurogenesis from postnatal neural stem cells. Nature. 2009;458:529-533.

35. Tsai CC, Su PF, Huang YF et al. Oct4 and Nanog directly regulate Dnmt1 to maintain selfrenewal and undifferentiated state in mesenchymal stem cells. Mol Cell. 2012;47:169182.

36. Bond AM, Bhalala OG, Kessler JA. The dynamic role of bone morphogenetic proteins in neural stem cell fate and maturation. Developmental neurobiology. 2012;72:1068-1084.

37. Panchision DM, McKay RD. The control of neural stem cells by morphogenic signals. Curr Opin Genet Dev. 2002;12:478-487.

38. Chen HL, Panchision DM. Concise review: bone morphogenetic protein pleiotropism in neural stem cells and their derivatives--alternative pathways, convergent signals. Stem Cells. 2007;25:63-68.

39. Kalyani AJ, Piper D, Mujtaba T et al. Spinal cord neuronal precursors generate multiple neuronal phenotypes in culture. J Neurosci. 1998;18:7856-7868.

40. Li W, Cogswell CA, LoTurco JJ. Neuronal differentiation of precursors in the neocortical ventricular zone is triggered by BMP. J Neurosci. 1998;18:8853-8862.

41. Shilatifard A. The COMPASS family of histone H3K4 methylases: mechanisms of regulation in development and disease pathogenesis. Annu Rev Biochem. 2012;81:6595.

42. Fang L, Zhang J, Zhang H et al. H3K4 Methyltransferase Set1a Is A Key Oct4 Coactivator Essential for Generation of Oct4 Positive Inner Cell Mass. Stem Cells. 2016;34:565-580.

43. Bledau AS, Schmidt K, Neumann $K$ et al. The H3K4 methyltransferase Setd1a is first required at the epiblast stage, whereas Setd1b becomes essential after gastrulation. Development. 2014;141:1022-1035.

44. Bi Y, Lv Z, Wang Y et al. WDR82, a key epigenetics-related factor, plays a crucial role in normal early embryonic development in mice. Biol Reprod. 2011;84:756-764. 
45. Yae T, Tajima K, Maheswaran S. SETD1A induced miRNA network suppresses the p53 gene expression module. Cell cycle (Georgetown, Tex). 2016;15:487-488.

46. Tajima K, Yae T, Javaid $\mathrm{S}$ et al. SETD1A modulates cell cycle progression through a miRNA network that regulates p53 target genes. Nature Communications. 2015;6:8257.

47. Hoshii T, Cifani P, Feng Z et al. A Non-catalytic Function of SETD1A Regulates Cyclin K and the DNA Damage Response. Cell. 2018;172:1007-1021.e1017.

48. Wu M, Wang PF, Lee JS et al. Molecular regulation of H3K4 trimethylation by Wdr82, a component of human Set1/COMPASS. Mol Cell Biol. 2008;28:7337-7344.

49. Lee JH, Skalnik DG. Wdr82 is a C-terminal domain-binding protein that recruits the Setd1A Histone H3-Lys4 methyltransferase complex to transcription start sites of transcribed human genes. Mol Cell Biol. 2008;28:609-618. 